\title{
Lipoma Gástrico. Lesión Submucosa Infrecuente. Reporte de Dos Casos Tratados
}

\author{
Unusual Gastric Submucosal Lipoma Lesion. Report of Two Cases
}

Carlos Manterola ${ }^{1,2}$ \& Carlos Ayala ${ }^{3}$ Luis Grande \& Tamara Otzen $^{2,5}$

\begin{abstract}
MANTEROLA, C.; AYALA, C.; GRANDE, L. \& OTZEN, T. Lipoma gástrico. Lesión submucosa infrecuente. Reporte de dos casos tratados. Int. J. Morphol., 36(3):1222-1224, 2018.

RESUMEN: El lipoma gástrico (LG), es un tumor benigno raro que representa el $5 \%$ de los lipomas gastrointestinales y menos del 1 $\%$ de todos los tumores gástricos. Se localizan habitualmente a nivel de la submucosa y generalmente en la región antral. La endoscopia y la tomografía computarizada son las herramientas diagnósticas más utilizadas en el proceso diagnóstico. Dada su relativa rareza, quedan a menudo fuera del diagnóstico diferencial de las masas submucosas gastrointestinales superiores. El objetivo de este manuscrito, es reportar dos casos tratados de forma consecutiva y revisar la literatura existente en esta materia. Se trata de dos pacientes de sexo masculino, de 49 y 69 años de edad, a quienes se diagnosticó una lesión submucosa gástrica por endoscopia y fueron tratados quirúrgicamente y cuyos especímenes fueron estudiados histológicamente, concluyéndose en ambos casos la existencia de un LG. Ambos pacientes evolucionaron de forma correcta y permanecen en controles clínicos y endoscópicos hasta la actualidad, sin inconvenientes. El LG es una entidad poco común, que puede simular una enfermedad maligna. Presentamos dos casos tratados quirúrgicamente con resultados satisfactorios.
\end{abstract}

PALABRAS CLAVE: Tumor gástrico benigno; Lipoma gástrico grande; Dolor abdominal superior.

\section{INTRODUCCIÓN}

Los lipomas gastrointestinales se observan principalmente en el yeyuno, íleon y colon. La localización gástrica de un lipoma, constituye una rareza; tanto así que, existe evidencia que representaría sólo el $5 \%$ de los lipomas gastrointestinales y menos del $1 \%$ de la totalidad de tumores gástricos (Cappell et al., 2017; Chagarlamudi et al., 2018). Los lipomas gástricos (LG), se localizan habitualmente en la submucosa; aunque puede surgir también de la capa subserosa o intramural (Cappell et al.). Su ubicación más frecuente es la región antral, siendo en su mayoría asintomáticos, en especial cuando son de tamaño pequeño. En el caso de los LG grandes ( $\geq 3 \mathrm{~cm}$ ), existe evidencia que apoya sus manifestaciones clínicas, entre las que destacan la hemorragia digestiva secundaria a la ulceración tumoral (Dargan et al., 2003; Kibria et al., 2009; Sadio et al., 2010; Ramdass et al., 2013; Almohsin \& Meshikhes, 2015; Krishnaraj et al., 2018; Yen et al., 2018), dispepsia (Nasa et al., 2016), obstrucción gástrica o pilórica (Furtado et al., 2013; Mehta et al., 2013; Rao et al., 2013; Priyadarshi et al., 2015), e incluso intususcepción tanto duodenal como yeyunal (Reza et al., 2018).
El objetivo de este manuscrito, es reportar dos casos tratados de forma consecutiva y revisar la literatura existente en esta materia.

\section{DESCRIPCIÓN DE LOS CASOS (Figs. 1 y 2)}

Caso 1: Se trata de un paciente de sexo masculino, de 69 años de edad, con antecedentes de hipertensión arterial en tratamiento; que fue derivado por una lesión gástrica submucosa de un año de evolución, diagnosticada por endoscopia; localizada en curva menor hacia cara posterior, de 2,5 cm de diámetro mayor. Se realizó endosonografía, que fue sugerente de GIST (spots y septos hiperecogénicos), de $26 \mathrm{~mm}$ de diámetro, localizado en la muscular propia. Por esta razón, se decide intervenirlo quirúrgicamente, lo que se lleva a efecto el 15/03/2018, oportunidad en la que se realiza una hemigastrectomía con gastroyeyuno anastomosis en Y-Roux. La histología del espécimen concluye "lipoma submucoso de curva menor, de $35 \mathrm{~mm}$ de diámetro”. El

\footnotetext{
${ }^{1}$ Departamento de Cirugía, Universidad de La Frontera Temuco Chile.

${ }^{2}$ Centro de Estudios Morfológicos y Quirúrgicos (CEMyQ), Universidad de La Frontera, Temuco, Chile.

${ }^{3}$ Clínica Mayor, Temuco Chile.

${ }^{4}$ Institut Hospital del Mar d'Investigacions Mèdiques (IMIM), Barcelona, España.

${ }^{5}$ Facultad de Ciencias de la Salud, Universidad de Tarapacá, Chile.

Financiado parcialmente por Proyecto MEC 80170022, CONICYT, Chile.
} 
paciente fue dado de alta al cuarto día, sin problemas. Cursando el $6^{\circ}$ mes postoperatorio, ha permanecido en controles clínicos y endoscópicos, con evolución satisfactoria.

Caso 2: Se trata de un paciente de sexo masculino, de 49 años de edad, con antecedentes de hipertensión arterial en tratamiento; que se encontraba en tratamiento por diverticulitis sigmoidea que deformaba el colon sigmoides. En la tomografía axial computarizada de control, se le pesquisa una lesión antral de $25 \mathrm{~mm}$, por lo que se solicita endoscopia. En ésta, se verifica la existencia de una lesión prepilórica de $25 \mathrm{~mm}$, que deforma el píloro. Se planificó para cirugía el 06/03/2018; oportunidad en la que se practicó colectomía izquierda con colo-recto anastomosis y exéresis por enucleación del tumor antral, cuya histología fue compatible con lipoma subseroso. El paciente fue dado de alta al cuarto día, sin problemas. En la actualidad, cursa su $9^{\circ}$ mes postoperatorio, con una evolución clínica y endoscópica normal.

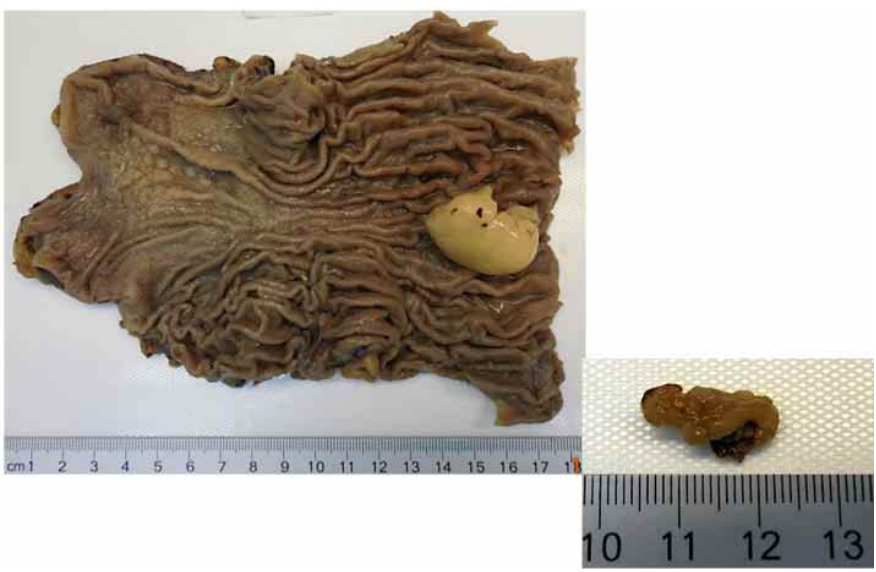

Fig. 1. Segmento de estómago de gastrectomía subtotal. Se aprecia mucosa con pliegues conservados parcialmente hacia la zona del píloro, donde destaca mucosa atrófica con áreas nodulares de 0,3 a $0,4 \mathrm{~cm}$. Hacia el margen proximal, se verifica una lesión polipoide y pediculada de $3,5 \times 2,5 \mathrm{~cm}$ de ejes mayores, ubicada a $1 \mathrm{~cm}$ de margen proximal.

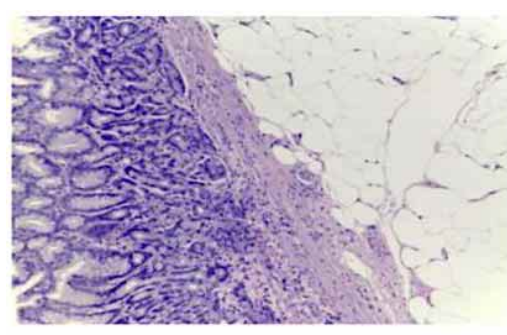

Fig. 2. Lesión pediculada constituida por adipocitos maduros, que presentan vacuola única citoplasmática que desplaza el núcleo. Se reconoce delicada trama capilar, sin signos inflamatorios.

\section{DISCUSIÓN}

El LG es un tumor benigno y poco frecuente, del tejido adiposo, que se rodea de una cápsula fibrosa y que aparece entre la $4^{\mathrm{a}}$ y $5^{\mathrm{a}}$ década de la vida (Cappell et al.). Habitualmente pasan desapercibidos o se confunden con tumores más frecuentes como GISTs, páncreas heterotópico, adenomiomas, leiomiomas, neurilemomas y fibromas; que son las lesiones con las que se plantea el diagnóstico diferencial (Neto et al., 2012). El primer reporte de LG conocido, pertenece a Jean Cruveilhier, quien lo describió en 1842 (Treska et al., 1998); y a pesar de ello, el número de casos reportados es muy escaso, de hecho, en una revisión sistemática publicada recientemente, se recuperaron sólo 32 casos de LG de gran tamaño $(\geq 4$ cm) desde 1980 (Cappell et al.).

Más del $90 \%$ se originan en la submucosa, localizándose preferentemente en la región antro pilórica, lo que permite explicar los casos de intususcepción al píloro o al duodeno, generando en síntomas dispépticos u obstructivos (Kim et al., 2011).

Desde el punto de vista endoscópico, suele verse como una masa submucosa, con o sin ulceración, lo que da la apariencia de "ojo de toro". También se pueden manifestar mediante los denominados signos de "tienda de campaña", "del gordo desnudo", y del "cojín" (Thompson et al., 2003; Kimura et al., 2005; Cappell et al.). Sin embargo, el diagnóstico diferencial con otras masas submucosas del estómago, como aquellas formadas por tejido muscular, nervioso, neuroendocrino; o por tumores estromales como los GIST; puede ser difícil (Kim et al., 2011). Por ello, es que se puede recurrir a la tomografía axial computarizada, la que permite apreciar lesiones circunscritas, de densidad grasa y con un valor de atenuación de -70 a -120 HU (Thompson et al.; Jeong \& Maeng, 2010; Kang et al., 2013; Lin et al., 2017; Ra et al., 2017); así como al ultrasonido de alta resolución, que permite visualizar la masa gástrica submucosa (Tsai et al., 2000).

El tratamiento de este tipo de lesiones es siempre quirúrgico (laparotómica, endoscópica o laparoscópica) y existe evidencia que sustenta la tesitura que la recurrencia postoperatoria es muy rara, pues se trata habitualmente de una lesión de carácter benigno (Dargan et al.; Jeong \& Maeng; Olguín et al., 2013; Matsushita et al., 2013; Lee et al., 2014).

El pronóstico de los LG corresponde al de un tumor benigno (Cappell et al.; Chagarlamudi et al.), sin recurrencias reportadas una vez extirpados. Sin embargo, existe evidencias de coexistencia con cáncer gástrico (Yamamoto et al., 2004; Namikawa et al., 2014). 


\author{
MANTEROLA, C.; AYALA, C.; GRANDE, L. \& OTZEN, T. \\ Unusual gastric submucosal lipoma lesion. Report of two cases. Int. \\ J. Morphol., 36(4):1222-1224, 2018.
}

SUMMARY: Gastric lipoma (GL), is a rare benign tumor that represents $5 \%$ of gastrointestinal lipomas and less than $1 \%$ of all gastric tumors. They are usually located at the level of the submucosa and usually in the antral region. Endoscopy and computed tomography are the diagnostic tools most used in the diagnostic process. Given their relative rarity, they are often left out of the differential diagnosis of the upper gastrointestinal submucosal masses. The aim of this manuscript was to report two cases treated consecutively and review the existing literature on this subject. Two male patients, 49 and 69 years of age, were diagnosed with a gastric submucosal lesion by endoscopy and were treated surgically. The specimens were studied histologically; concluding the existence of a GL in both cases. The two patients evolved adequately and have remained in clinical and endoscopic controls until now, without problems. GL is a rare entity, which can simulate a malignant disease. We present two cases treated surgically with satisfactory results.

KEY WORDS: Benign gastric tumor; Large gastric lipoma; Upper abdominal pain.

\section{REFERENCIAS BIBLIOGRÁFICAS}

Almohsin, M. \& Meshikhes, A. W. Gastric lipoma presenting with haematemesis. BMJ Case Rep., 2015, 2015. doi:10.1136/bcr-2014-206884.

Cappell, M. S.; Stevens, C. E. \& Amin, M. Systematic review of giant gastric lipomas reported since 1980 and report of two new cases in a review of 117110 esophagogastroduodenoscopies. World J. Gastroenterol., 23(30):5619-33, 2017.

Chagarlamudi, K.; Devita, R. \& Barr, R. G. Gastric lipoma: a review of the literature. Ultrasound Q., 34(3):119-21, 2018.

Dargan, P.; Sodhi, P. \& Jain, B. K. Bleeding gastric lipoma: case report and review of the literature. Trop. Gastroenterol., 24(4):213-4, 2003.

Furtado, W. S.; Mello, D. A.; Santos, V. M.; Bringel, T. L.; Oliveira Junior, W. P. \& Moura, H. J. Gastric lipoma and pyloric obstruction in a 51-year-old woman. An. Sist. Sanit. Navar., 36(1):145-8, 2013.

Jeong, I. H. \& Maeng, Y. H. Gastric lipomatosis. J. Gastric Cancer, 10(4):254$8,2010$.

Kang, H. C.; Menias, C. O.; Gaballah, A. H.; Shroff, S.; Taggart, M. W.; Garg, N. \& Elsayes, K. M. Beyond the GIST: mesenchymal tumors of the stomach. Radiographics, 33(6):1673-90, 2013.

Kibria, R.; Butt, S.; Ali, S. A. \& Akram, S. An unusual case of giant gastric lipoma with hemorrhage. J. Gastrointest. Cancer, 40(3-4):144-5, 2009.

Kim, D. D.; Tsai, A. I. A.; Otani, A. R. O.; Puglia, C. R. \& Malheiros, C. A. Lipoma gástrico: relato de caso. Rev. Col. Bras. Cir., 38(3):205-6, 2011.

Kimura, H. E. T.; Vázquez-Lamadrid, J. \& Téliz-Meneses, M. A. Tumores estromales del tubo digestivo: Experiencia radiológica del Instituto Nacional de la Nutrición. An. Radiol. Méx., 4(3):255-69, 2005.

Krishnaraj, B.; Dhanapal, B.; Shankar, G.; Sistla, S. C.; Galidevara, I. \& Suresh, A. Gastric lipoma: a rare cause of haematemesis. Ann. R. Coll. Surg. Engl., 100(3):e41-3, 2018.

Lee, K. J.; Kim, G. H.; Park, D. Y.; Shin, N. R.; Lee, B. E.; Ryu, D. Y.; Kim, D. U. \& Song, G. A. Endoscopic resection of gastrointestinal lipomas: a single-centerexperience. Surg. Endosc., 28(1):185-92, 2014.

Lin, Y. M.; Chiu, N. C.; Li, A. F.; Liu, C. A.; Chou, Y. H. \& Chiou, Y. Y. Unusual gastric tumors and tumor-like lesions: Radiological with pathological correlation and literature review. World J. Gastroenterol., 23(14):2493-504, 2017.
Matsushita, M.; Fukata, N. \& Okazaki, K. Endoscopic removal of large gastric lipomas: en bloc resection with submucosal dissection or partial resection with unroofing technique? Dig. Endosc., 25(2):211-2, 2013.

Mehta, J. A.; Khedkar, K. B.; Thakur, V. V.; Singh, R. \& Joshi, R. M. Gastric antral lipoma presenting as gastric outlet obstruction. Indian J. Gastroenterol., 32(6):422, 2013.

Namikawa, T.; Munekage, E.; Mizuta, H.; Kobayashi, M.; Saibara, T. \& Hanazaki, K. Simultaneous occurrence of gastric lipoma and early gastric cancer. Endoscopy, 46 Suppl. 1 UCTN:E338-9, 2014.

Nasa, M.; Choksey, A.; Phadke, A. \& Sawant, P. Gastric lipoma: an unusual cause of dyspeptic symptoms. BMJ Case Rep., 2016, 2016. doi: 10.1136/ bcr-2016-215297.

Neto, F. A.; Ferreira, M. C.; Bertoncello, L. C.; Neto, A. A.; de Aveiro, W. C.; Bento, C. A.; Cecchino, G. N. \& Rocha, M. A. Gastric lipoma presenting as a giant bulging mass in an oligosymptomatic patient: a case report. $J$. Med. Case Rep., 6:317, 2012.

Olguín, R. R.; Norero, M. E.; Briceño, E.; Martínez, C.; Viñuela, E.; Báez, S.; Aguayo, G.; Calvo, A.; Mege, R. R. \& Díaz, A. Gastric lipoma removed by laparoscopic subtotal gastrectomy: report of one case. Rev. Med. Chil., 141(7):927-31, 2013

Priyadarshi, R. N.; Anand, U.; Pandey, M. K.; Chaudhary, B. \& Kumar, R. Giant gastric lipoma presenting as gastric outlet obstruction - A case report. J. Clin. Diagn. Res., 9(10):PD03-4, 2015.

Ra, J. C.; Lee, E. S.; Lee, J. B.; Kim, J. G.; Kim, B. J.; Park, H. J.; Park, S. B. \& Choi, B. I. Diagnostic performance of stomach CT compared with endoscopic ultrasonography in diagnosing gastric subepithelial tumors. Abdom. Radiol. (N. Y.), 42(2):442-50, 2017.

Ramdass, M. J.; Mathur, S.; Seetahal-Maraj, P. \& Barrow, S. Gastric lipoma presenting with massive upper gastrointestinal bleeding. Case Rep. Emerg. Med., 2013:506101, 2013.

Rao, C.; Rana, S. S.; Lal, A.; Kumar, M.; Behera, A.; Dahiya, D.; Joshi, K. \& Bhasin, D. K. Large gastric lipoma presenting with GI bleeding. Gastrointest. Endosc., 77(3):512-3, 2013.

Reza, J. A.; Fruchter, S.; Varadarajulu, S. \& Arnoletti, J. P. A large intussuscepting gastric lipoma. J. Gastrointest. Surg., 22(7):1299-300, 2018.

Sadio, A.; Peixoto, P.; Castanheira, A.; Cancela, E.; Ministro, P.; Casimiro, C. \& Silva, A. Gastric lipoma--an unusual cause of upper gastrointestinal bleeding. Rev. Esp. Enferm. Dig., 102(6):398-400, 2010.

Thompson, W. M.; Kende, A. I. \& Levy, A. D. Imaging characteristics of gastric lipomas in 16 adult and pediatric patients. A. J. R. Am. J . Roentgenol., 181(4):981-5, 2003.

Treska, V.; Pesek, M.; Kreuzberg, B.; Chudácek, Z.; Ludvíková, M. \& Topolcan, O. Gastric lipoma presenting as upper gastrointestinal obstruction. $J$. Gastroenterol., 33(5):716-9, 1998

Tsai, T. L.; Changchien, C. S.; Hu, T. H. \& Hsiaw, C. M. Demonstration of gastric submucosal lesions by high-resolution transabdominal sonography. J. Clin. Ultrasound, 28(3):125-32, 2000.

Yamamoto, T.; Imakiire, K.; Hashiguchi, S.; Matsumoto, J.; Kadono, J.; Hamada, N.; Yoshioka, T. \& Kitajima, S. A rare case of gastric lipoma with early gastric cancer. Intern. Med., 43(11):1039-41, 2004.

Yen, H. H.; Lin, K. H. \& Chen, C. J. Gastrointestinal: Gastric lipoma as a rare cause of recurrent gastrointestinal bleeding. J. Gastroenterol. Hepatol., 33(4):771, 2018

Dirección para correspondencia:

Carlos Manterola

Departamento de Cirugía y CEMyQ

Universidad de La Frontera

Manuel Montt 112, Oficina 408

Temuco

CHILE

Recibido : 20-04-2018

Aceptado: 19-06-2018

Email: carlos.manterola@ufrontera.cl 